Reporte de caso

\title{
Linfangioma en maxilar de un recién nacido: Reporte de un caso clínico.
}

Sebastián Eduardo Miguelez ${ }^{1}$; Lorena Gonzalez 2; Ariel Monteagudo ${ }^{1}$; Christian Oscar Mosca ${ }^{3}$;

\section{Resumen}

Los linfangiomas son neoplasias benignas que ocurren predominantemente en la infancia, son muy infrecuentes, y nacen del sistema linfático. Cuando ocurren en la cavidad oral, la localización más común es el dorso de la lengua, seguido por los labios, la mucosa bucal, el paladar blando y el piso de la boca. La prevalencia es de 1 a $3 / 10.000$ nacidos vivos, afectando ambos generos por igual, involucran en un $75 \%$ la cabeza y el cuello, seguido por el tronco, abdomen y extremidades. En esta publicación presentamos una situación de una paciente de sexo femenino de menos de 24 horas de vida que reside en el Servicio de Neonatología H.I.G.A. Pte. Perón en la que se decidió realizar la exéresis de la patología. El objetivo del tratamiento instaurado fué la remoción del tejido exofítico, con margen de seguridad del mismo debido al pequeño tamaño y su base pediculada. Dicho material fué remitido a la Cátedra de Anatomía Patológica de la Facultad de Odontología de la Universidad de Buenos Aires y su diagnóstico histopatológicos, morfológico e inmunohistoquímicos comprobaron la compatibilidad de linfangioma.

A los 18 meses de seguimiento post operatorio la paciente no muestra signos evidentes de recidiva o asociados a la patología diagnosticada.

Palabras Clave: Linfangioma; Recien nacido; Neoplasia.

\footnotetext{
${ }^{1}$ Odontólogo del Hospital Interzonal General de Agudos Gral Perón. Buenos Aires, Argentina.

${ }^{2}$ Jefe de Trabajos Prácticos. Cátedra de Cirugía y Traumatología Buco Maxilofacial I Facultad de Odontología. Universidad de Buenos Aires. Buenos Aires, Argentina.

${ }^{3}$ Jefe de Trabajos Prácticos. Cátedra de Cirugía y Traumatología Buco Maxilofacial II. Cátedra de Microbiología y Parasitología. Facultad de Odontología. Universidad de Buenos Aires. Buenos Aires, Argentina.
} 
Relato de caso

\section{Linfangioma em queixada de um recém-nascido: relato de um caso.}

\section{Resumo}

Os Linfangiomas são neoplasias benignas que acontecem geralmente na infância, são muito frequentes, e se originam no sistema linfático. Quando ocorre na cavidade oral, a localização mais comum é no dorso da língua, seguida pelos lábios, mucosa bucal, palato mole e o assoalho da boca. A prevalência é de 1 a $3 / 10.000$ nascidos vivos, afetando ambos gêneros igualmente. Cerca 75\% acometem região de cabeça e pescoço, seguida pelo tronco, abdômen e membros. Nesta publicação, vamos apresentar uma situação de um paciente do sexo feminino de menos de 24 horas de vida proveniente do Ser- viço de Neonatologia H.I.G.A. Pte. Peron, onde se decidiu fazer a exérese da lesão. O objetivo do tratamento foi a remoção do tecido exofítico, com margem de segurança, devido ao tamanho pequeno e sua base pediculada. $\mathrm{O}$ material foi enviado à Cadeira de Anatomia Patológica da Faculdade de Odontologia da Universidade de Buenos Aires e seu diagnóstico histopatológico, morfológico e imunohistoquímico confirmou a compatibilidade com linfangioma. Aos 18 meses de seguimento pós-operatório, o paciente não apresentou sinais evidentes de recidiva ou associados à patologia diagnosticada.

Palavras chave: Linfangioma; recém nascido; Neoplasia.

Case report

\section{Maxilar lymphangioma in a newborn patient: A case report.}

\begin{abstract}
Lymphangiomas are benign neoplasms that appear preferentially in childhood, are very uncommon, and have their origin in the lymphatic system. When they occur in the oral cavity, the most frequent location is the back of the tongue, followed by lips, buccal mucosa, soft palate and floor of the mouth. Prevalence is $1-3 / 10,000$ live births, equally affecting both genders, with $75 \%$ involving the
\end{abstract}

head and neck region followed by trunk, abdomen, and extremities. In this publication we present the case of a female patient less than 24 hours of life that remained in the Service of Neonatology Service of H.I.G.A Pte. Perón. According to the clinical evaluation of the Dentistry Service it was decided to perform the excisional biopsy of the pathology. The aim of the treatment was to remove of the exophytic tissue, with safety margin due to the small size and pediculated base. This 
material was sent to the Chair of Pathological Anatomy of the Odontology Faculty of the University de Buenos Aires and his histopathological, morphological and immunohistochemical diagnosis proved the compatibility of lymphangioma. At the 18 months

\section{Introducción}

Los linfangiomas son neoplasias benignas, pero pueden actuar como neoplasias malignas al infiltrarse a estructuras vitales e invadir tejidos. Estas lesiones ocurren predominantemente en la infancia, son muy infrecuentes, y nacen del sistema linfático de la piel del cuello, axila, mediastino y retroperitoneo, siendo su localización más frecuente cabeza y cuello, ${ }^{1,2,3}$ pero pudiéndose desarrollar en cualquier órgano o tejido del cuerpo. $^{2,4,5}$

El Linfangioma fue descrito por primera vez por Redenbacher en $1828^{6}$ y linfangiomas de la lengua fue descrito por primera vez por Virchow en $1854^{7}$.

Teniendo en cuenta que la incidencia de malformaciones congénitas que afectan el cuello, los labios y el paladar es relativamente baja. Dentro de estas lesiones, se incluyen básicamente las congénitas, las inflamatorias y las neoplásicas; los linfangiomas son los más comunes dentro de las lesiones congénitas $^{8,9}$ ya que ocupan el $30 \%$ de todas las malformaciones encontradas por ecografía prenatal. ${ }^{10}$

Cuando ocurren en la cavidad oral, la localización más común es el dorso de la lengua, seguido por los labios, la mucosa bucal, el paladar blando y el piso de la boca ${ }^{11}$. Clínicamente, los linfangiomas son de crecimiento lento, la masa de tejido blando sin dolor. La apariencia clínica del linfangioma depende de la extensión de post-operative follow-up, the patient did not show obvious signs of relapse or othe complications associated with the diagnosed pathology.

Keywords: Lymphangioma; Newborn; neoplasms.

la lesión. Las lesiones superficiales consisten en nódulos elevados de color rosado o amarillento o vistos como vesículas agrupadas transparentes, que pueden ser de color rojo o púrpura debido a hemorragias secundarias. ${ }^{9}$ Las lesiones más profundas se describen como masas suaves y difusas con color normal. ${ }^{11}$

Morfológicamente se clasifican en tres tipos:2,4 linfangioma simple formado por pequeños vasos linfáticos de paredes delgadas, linfangioma cavernoso constituido por vasos linfáticos de pequeño tamaño con capas conectivas de grosor irregular entre ellos, y linfangiomas o higroma quístico cuando está compuesto por grandes espacios linfáticos macroscópicos que poseen revestimiento de colágeno y músculo liso4. Kennedy y Cols. los clasifican en linfagiomas simples, cavernosos, higroma quístico y linfagioma difuso. ${ }^{12}$

El sistema de clasificación más relevante es el que tiene en cuenta el tamaño de estas lesiones, como macroquístico (quistes mayores que $1 \mathrm{~cm}$ ), microcística (cuando la mayoría de los quistes son más pequeños que $1 \mathrm{~cm}$ ), o mezclado. Se debe esta clasificación porque los linfangiomas microcísticos son menos sensible a los tratamientos clínicos como la escleroterapia. ${ }^{13}$

Un linfangioma o higroma quístico ocurre a medida que el bebé crece en el útero. Se forma a partir de fragmentos de material que lleva líquido y glóbulos blancos. Dicho material se deno- 


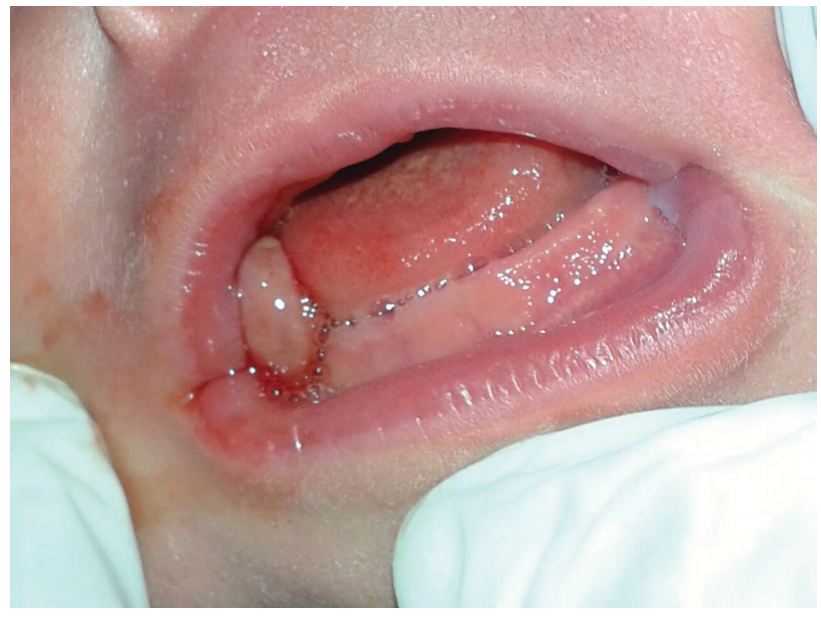

Figura 1. Masa de $2 \times 1 \times 1 \mathrm{~cm}$. de tejido blando, en zona reborde de paladar, altura 54 y 55 .

mina tejido linfático embrionario. Después del nacimiento, un higroma quístico con frecuencia luce como una protuberancia suave bajo la piel. El quiste tal vez no se detecte al nacer. Típicamente crece conforme crece el niño. Algunas veces, no se nota hasta que el niño es más grande. ${ }^{4}$

El objetivo del tratamiento instaurado fue la remoción del tejido exofítico, con margen de seguridad del mismo debido al pequeño tamaño y su base pediculada y un control muy exhaustivo de la patología al mes, a los 3 meses, 12 meses, encontrándose el paciente en la actualidad en el control de los 18 meses.

\section{Materiales y métodos}

Para la presente investigación fundamentalmente se protegieron los derechos del paciente, primeramente bajo el consentimiento firmado por la madre del representado y la autorización en el área de docencia del Hospital Interzonal de Agudos Gral Perón, respetando los principios éticos basado en la Declaración de Helsinki, la presente situación fue sometida a consideración por el comité de bioética del Hospital Interzonal de Agudos Gral Perón, bajo Aval en fecha 7 de julio de 2017.

\section{Diagnóstico por imágenes: Situacion clinica}

Se recibe interconsulta, del Servicio de Neonatología H.I.G.A. Pte. Perón. Paciente de sexo femenino de menos de 24 horas de vida. A la inspección clínica se observaba una masa de $2 \times 1 \times 1$ $\mathrm{cm}$. de tejido blando, color rosa coral, a la palpación de consistencia firme aunque algo depresible en zona de paladar altura 54 y 55. (Fig 1)

Al quinto día de vida, el crecimiento de la masa comenzó a impedir la alimentación, por lo que el cuerpo médico del Servicio de Neonatología, decidió implementar la alimentación por vía parenteral. Es por tal motivo que al dia siguiente se decidió realizar la exeresis de la patología y posterior envío para su examen histopatológico.

Se realizó punción aspirativa de la masa tumoral, en la que se observó leve cantidad de contenido serohemático, de color parduzco. Al que se lo envió a cultivar.

\section{Técnica quirúrgica}

Con el objetivo de disminuir la cantidad y actividad de la microbiota bucal, se realizó antisepsia por fricción en la zona del abordaje quirúrgico con solución de yodo povidona al $10 \%$. Posteriormente se anestesió con una infiltrativa en fondo de surco y paladar (Fig 2). Se realizaron maniobras quirúrgicas pertinentes para acceder a la entidad patológica realizando un punto tractor, para luego con bisturí frio hoja $\mathrm{N}^{\circ} 15 \mathrm{c}$ realizar la incisión inmediatamente por debajo del punto 


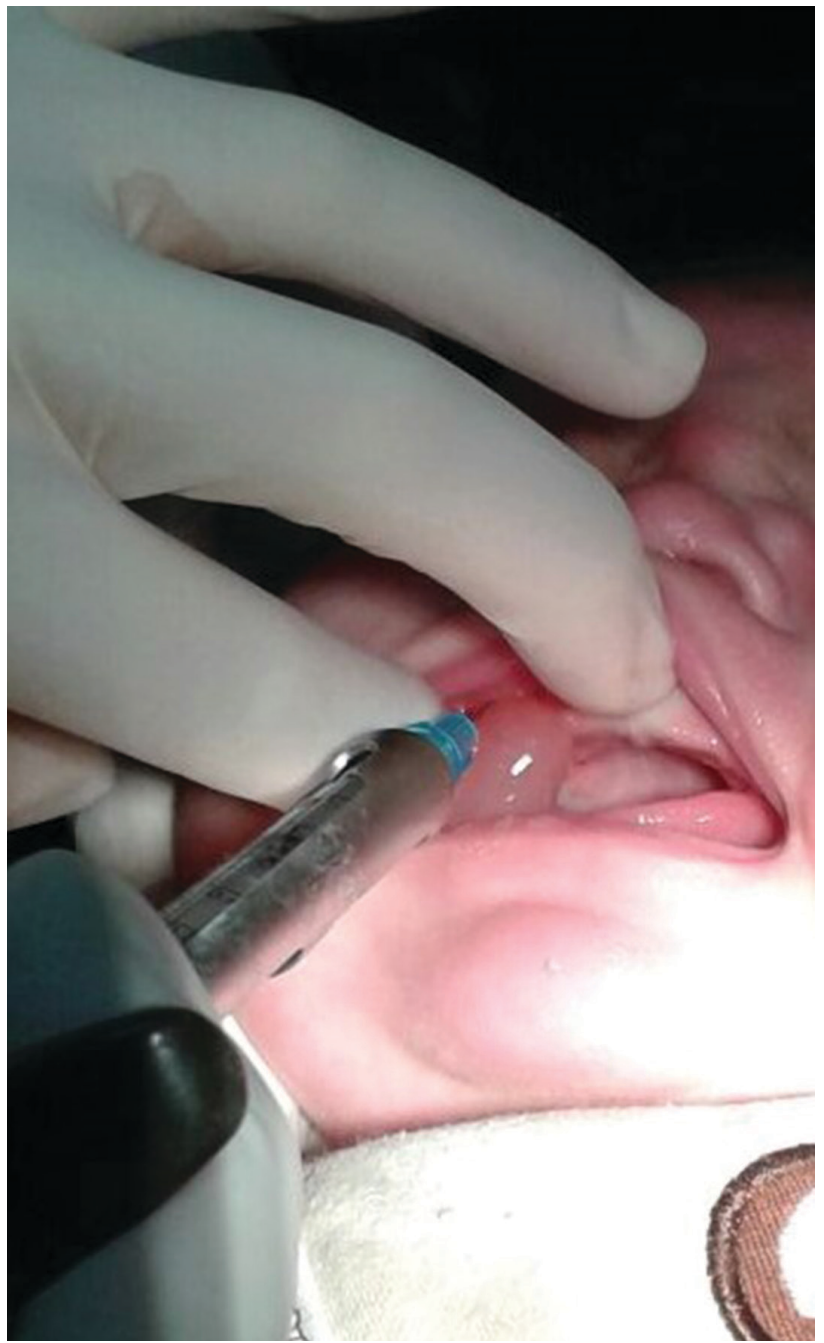

Figura 2. Anestesia local con epinefrina en fondo de surco y paladar.

realizado (Fig 3 y 4). La pieza resecada se sumergió en formol al 10\% y se envió a la Cátedra de Anatomía Patológica de la Facultad de Odontología de Universidad de Buenos Aires (Fig 5).

\section{Estudios anatomopatológicos:}

Al examen macroscópico mostro una formación lobulada de 2x1x1 cm, con cubierta mucosa epitelial lisa de color beige. Consistencia al corte gomosa.

Al examen microscópico presentaba multiples canales vasculares de tipo linfático que se anas-

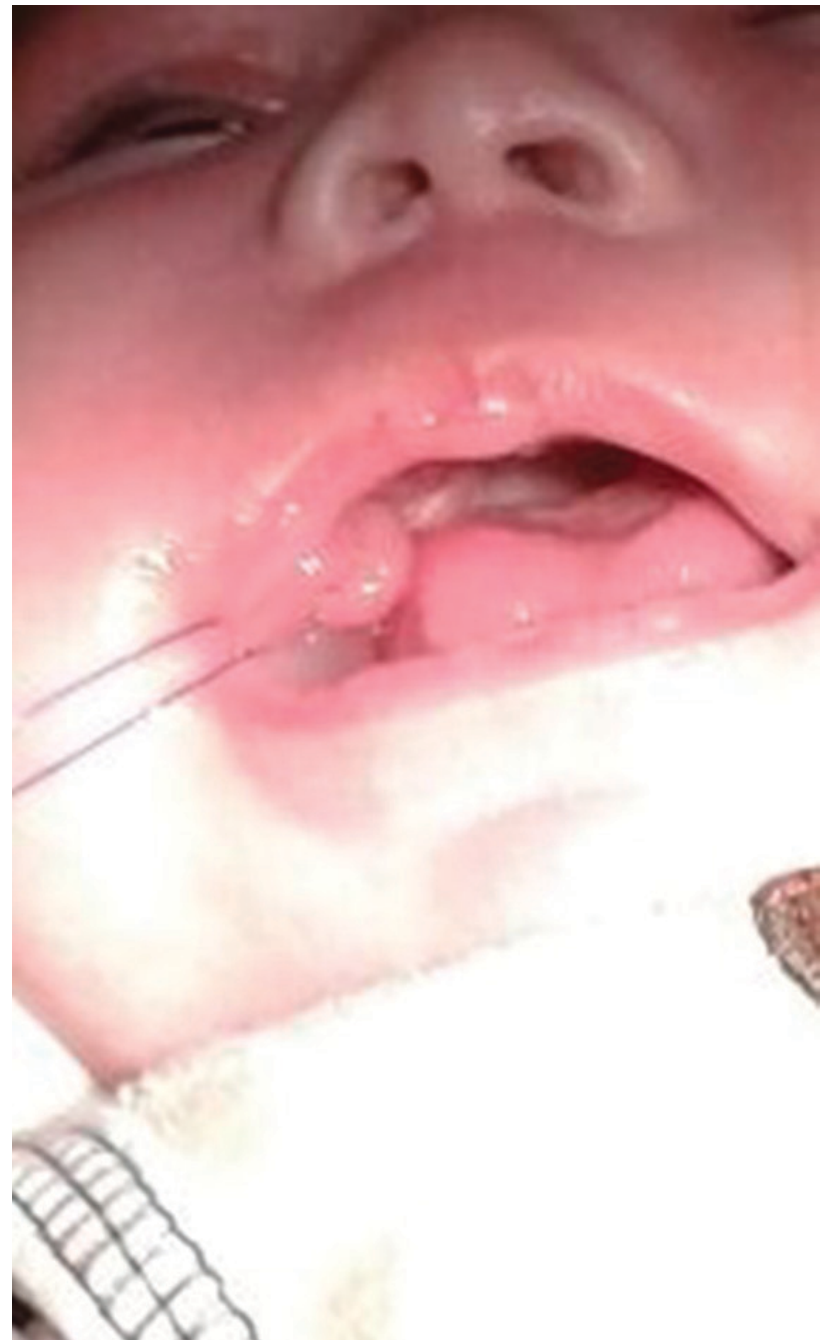

Figura 3. Punto de tracción.

tomosan formando plexos, los mismos se encontraban tapizados por una mono capa de células endoteliales aplanadas que en sectores mostraban estructuras de aspecto papilar (Fig 6).

Se le realizaron estudios de inmunohistoquimica de CD 34; Podoplanin (D2 40); Miogenina y Desmina. Los resultados a estoy estudios fueron negativos a excepción del Podoplanin que dio resultado positivo.

Sobre la base de los estudios morfológicos y el perfil inmunológico se vinculó la patología a un Linfangioma. 


\section{Controles Post Quirúrgicos}

A los 10 días posteriores de la intervención se la citó para control post quirúrgico. Luego se la evaluó al mes, a los 3 meses, 12 meses y 18 meses. Se evidencio una pequeña cicatriz en el área quirúrgica.

$\mathrm{Al}$ control realizado a los 3 meses posquirúrgicos, la madre refiere la aparición de una mancha color rojiza, levemente elevada, en cuero cabelludo. La lesión se encontraba a nivel de la fontanela fronto-parietal, a la inspección clínica

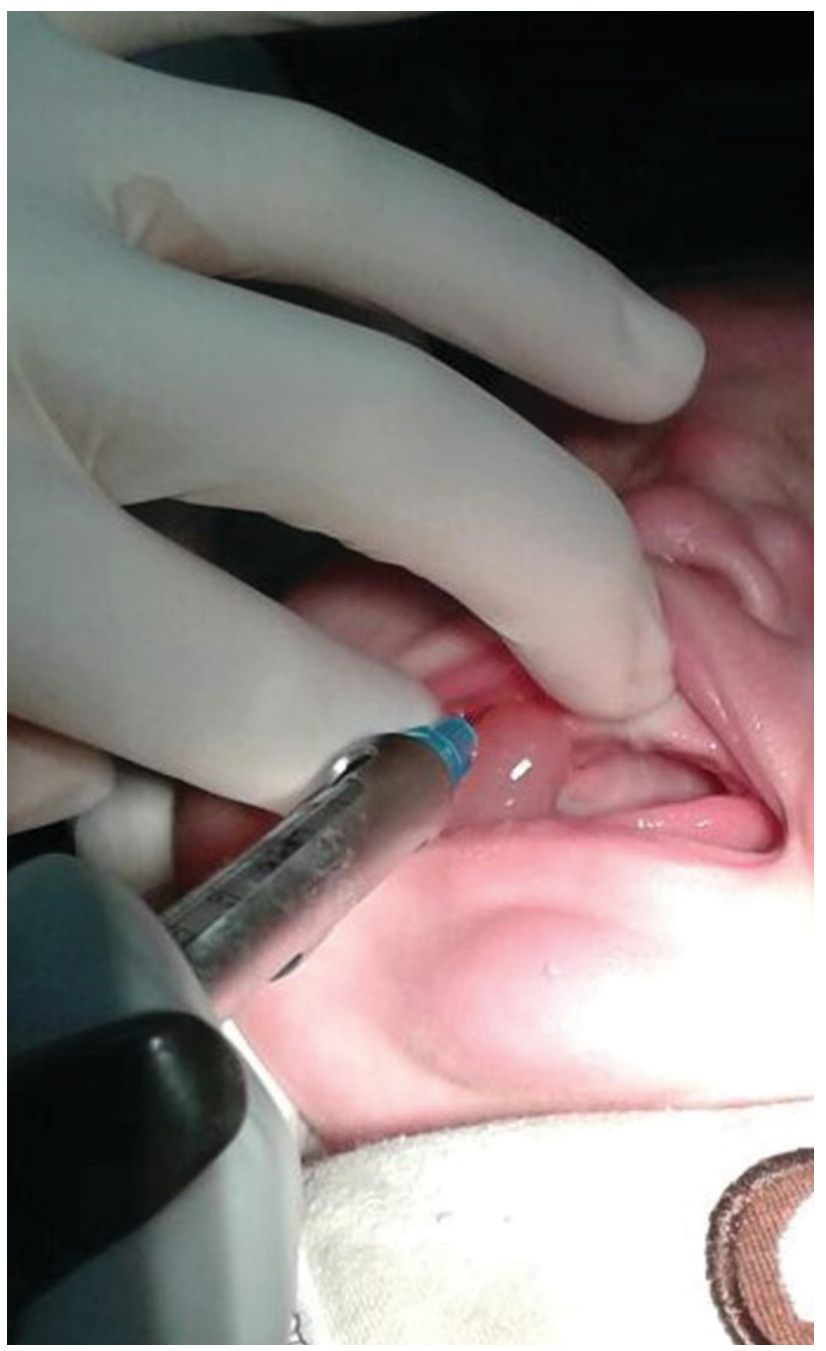

Figura 4. Lesión extirpada. Se puede observar la dimensión de la masa tumoral.

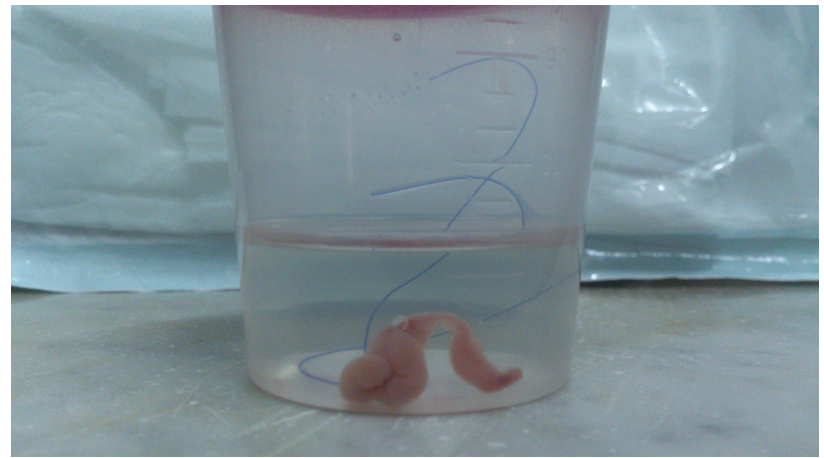

Figura 5. Remisión para su análisis Anatomopatológico.

la patología en cuestión se compatibiliza con un hemangioma, el cual no ha mostrado modificaciones en controles posteriores (Fig 7).

Debido a la posibilidad de recidiva, la paciente continúa en controles trimestrales, siendo el último control a los 18 meses de vida (Fig 8). En el mismo se puede observar una hiperplasia cicatrizal de unos 2 a $3 \mathrm{~mm}$ de diámetro, que siempre se mantuvo igual en todos los controles postquirúrgicos, y nos encontramos evaluando la erupción de las piezas 54 y 55.

\section{Discusion}

Los linfangiomas aparecen con mayor frecuencia en niños menores de 1 año, 80-90\% se manifiestan antes de los 2 primeros años de vida, desciende ligeramente su incidencia hasta los 5 años, y progresivamente disminuye hasta los 16 años. ${ }^{14,15}$ La prevalencia es de 1/12.000 nacimientos y se observa con más frecuencia en el sexo masculino. ${ }^{16,17}$ Son localizados preferentemente en cabeza y cuello. Pueden ser cavernosos y/o quísticos, y están revestidos por endotelio aplanado, con tabiques fibroconectivos, músculo liso y nódulos linfoides. El contenido es linfa y/o sangre. Se asocian cambios degenerativos de tipo mixoide, inflamatorio, hemorrágico o isquémico. Las com- 

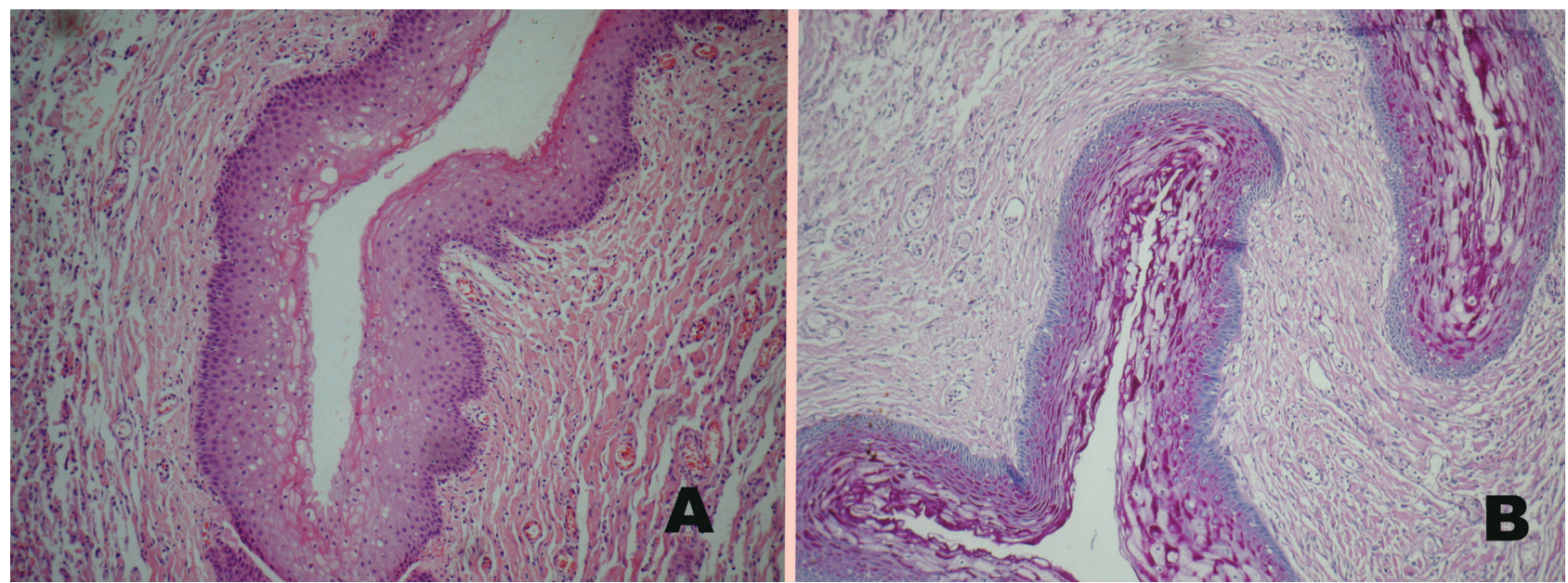

Figura 6. En la microscopia del preparado anatomopatológico (a. eosina-hematoxilina y b. técnica de PAS) se puede observar un epitelio pavimentoso estratificado paraqueratinizado. Con un corion levemente denso en que se visualizan fibras colágenas delicadas con presencia de fibroblastos entre las mismas. Presenta múltiples canales vasculares de tipo linfático que se anastomosan formando plexos.

plicaciones que producen se deben también al tamaño que alcanzan y a la localización. El 6\% de los linfangiomas recidivan asociado al componente cavernoso microscópico que muestran. El patólogo tiene la misión de aportar el diagnóstico de la lesión, extensión y valoración de bordes quirúrgicos. El diagnóstico diferencial de linfangioma incluye el número de lesiones orales tales como hemangioma, teratoma, quiste dermoide, quiste de conducto tirogloso, amiloidosis, neurofibromatosis, heterotopía de quistes mucosos gástricos y tumor de células granulares.

Esta entidad son malformaciones congénitas del sistema linfático; su origen no está definido. Se cree que se inician en la sexta semana de gestación, formándose 2 sacos yugulares que empiezan como hendiduras en el mesénquima cervical, y de ahí derivan 3 teorías donde estas hendiduras fallan al conectarse y forman canales de linfa, ocasionándose un inadecuado drenaje al sistema venoso, lo cual los dilata. Otra teoría postula que son el resultado de depósitos de tejido linfático en áreas equivocadas duran- te la embriogénesis y, por tanto, una alteración en la unión hacia el sistema linfático normal. La última teoría es una construcción anormal del sistema linfático, lo que hace que no haya una conexión con los canales linfáticos centrales. ${ }^{18}$

Cuando la cavidad oral está afectada, puede incluir la lengua, el paladar, la mucosa gingival y oral, los labios y la cresta alveolar de la mandíbula. Brennan y Cols., ${ }^{19}$ revisaron 49 casos de linfangioma oral y encontraron que 17 se localizaban en lengua mientras que sólo se describía un caso en la región retromolar y uno en el paladar blando. En el presente caso la patología se encontraba maxilar superior sobre el reborde alveolar altura de los molares con un leve desplazamiento hacia palatino Mismo la lesión no dejaba que el recién nacido pueda nutrirse por lo que había dejado de amamantar.

En los niños no es raro el hallazgo de un linfangioma tras extirpación de una glándula salival por el diagnóstico de tumor o quiste de retención salival, ya que los tumores vasculares son frecuentes en las mismas, tanto intraglandula- 


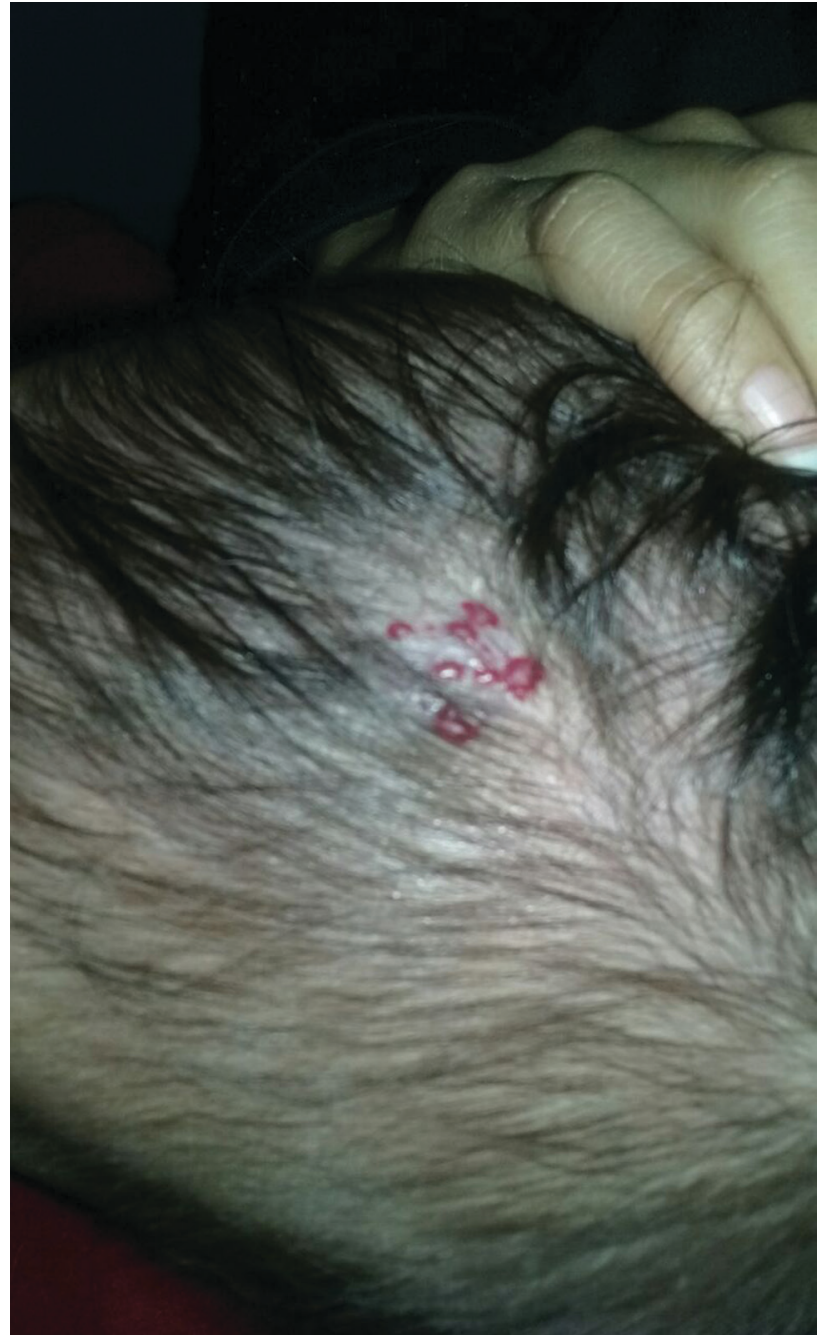

Figura 7. Hemangioma en cuero cabelludo a los 3 meses de vida.

res, como las afectadas por extensión de un linfangioma de cuello. ${ }^{20}$

En el estudio realizado por Jorgenson en el año 1987 la incidencia recaía en en neonatos de raza negra, con un ratio de incidencia del 3,6\% . Friend observó un aumento de la incidencia de este tipo de lesiones si las madres de los niños eran adolescentes. ${ }^{21}$

Aún se indica la extirpación completa de la lesión como el tratamiento más aceptado y de primera elección ${ }^{22}$, aunque hay una evidencia del $10-39 \%$ de recurrencias. ${ }^{23,}{ }^{24}$ La recidiva es común debido a su naturaleza infiltrativa.

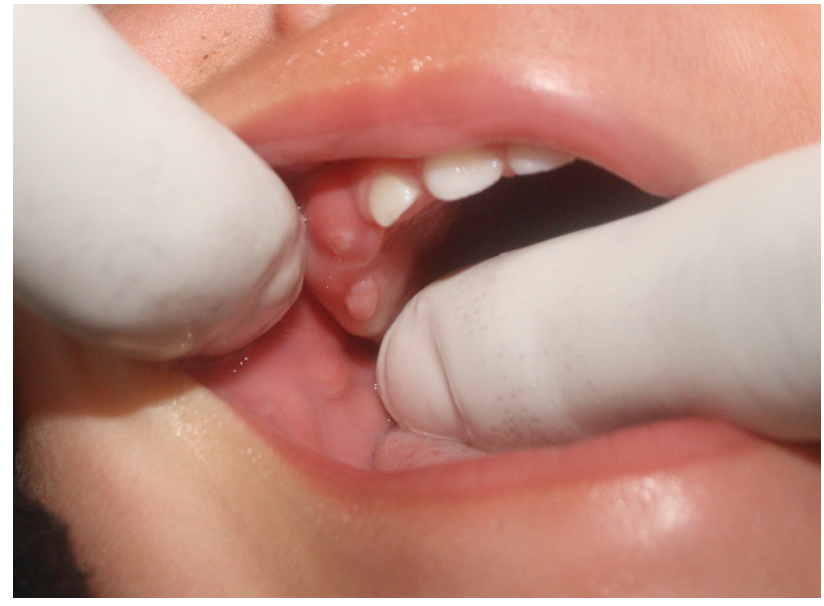

Figura 8. Control post quirúrgico a los 18 meses de vida. Se puede observar una hiperplasia cicatrizal de aproximadamente 2 a 3 mm de diámetro. La misma mantiene sus dimensiones en todos los respectivos controles.

Con las limitaciones de la cirugía, existen autores que describen la terapia exclerosante como alternativa, con el objetivo de obtener la regresión total o parcial del linfangioma, sin embargo, pueden aparecer efectos adversos, como fibrosis pulmonar con el uso de bleomicina y la consecuente muerte; con dextrosa al $50 \%$ policresuleno también han aparecido recidivas, aunque en menor medida que con el tratamiento quirúrgico. ${ }^{25,26}$

En algunos países, incluyendo la Argentina, se utiliza un tratamiento llamado OK 432 que es un agente inmunomodulador, producto de la liofilizacion del cultivo mixto de la cepa, su de baja virulencia del Streptococcus pyogenes, grupo A, tipo $111 \mathrm{~B}$ de origen humano, el cual ha sido incubado con penicilina $G$ potásica, por lo que no puede usarse en niños con alergia a penicilina. Este producto actúa sobre la malformación congénita a través de una cascada de agentes antitumorales secretados por neutrófilos, macrófagos, linfocitos natural killer (CD56+) y células T (CD3+), que activan el sistema inmune ocasionando un incremento de la permeabilidad endotelial, del drenaje y del 
flujo linfático, dando como resultado la reducción de la lesión por efecto de la contracción de los espacios quísticos.27 $\mathrm{El} \mathrm{OK} 432$ tiene uso restringido en los Estados Unidos.

\section{Conclusión}

Teniendo en cuenta que las terapias esclerosantes presentan un riesgo por sus efectos adversos descritos por varios autores, y basándonos en la literatura en donde todavía se indica la extirpación completa de la lesión como el tratamiento más aceptado y de primera elección a pesar del porcentaje de recidiva; optamos por la extirpación quirúrgica con un control muy exhaustivo de la misma. En nuestro caso al ser un recién nacido con una lesión con base pediculada, la misma nos permitió un margen de seguridad sin complicaciones quirúrgicas bajo anestesia local. Después de 18 meses de seguimiento, no se identificó ningún signo de recidiva de la lesión.

\section{Referencias bibliográficas}

1. Stal S, Hamilton S, Spira M. Hemangiomas, lymphangiomas and vascular malformation of the head and neck. Otolaringol Clin North Am 1986; 19: 769-796.

2. Hilliard R, McKendry J, Phillips MJ. Congenital abnormalities of the lymphatic system: A new clinical classification. Pediatrics 1990; 86: 988-994.

3. Fattahi S, Vosoughhosseini S, Moradzadeh Khiavi M, Mostafa Mahmoudi S, Emamverdizadeh P, Gholamreza Noorazar

S, Yasamineh N, Lotfi R. Prevalence of Head and Neck Tumors in Children under 12 Years of Age Referred to the Pathology Department of Children's Hospital in Tabriz during a 10-year Period. Journal of Dental Research, Dental Clinics, Dental Prospects Vol. 9, No. 2 Spring 2015.

4. Enzinger FM, Weiss SW. Soft tissue tumores, 2ª edition. St. Louis: C.V. Mosby Company, 1998; 614-637.

5. Bansal N, Haidar-El-Atrache S, Walters H, Kobayashi D. Cardiac Lymphangioma Encasing Right Coronary Artery in an Infant. Ann Thorac Surg 2017; 104: e279-81.

6. Coffin CM, Dehner LP. Vascular tumours in childrenand adolescents: A clinicopathologic study of 228 tumoursin 222 patients. Pathol Annu. 1993; 28: 97-120.

7. Balakrishnan A, Bailey CM. Lymphangioma of the tongue. A review of pathogenesis, treatment and the use of surface laser photocoagulation. J Laryngol Otol. 1991; 105: 924-9.

8. Brown RL, Azizkhan RG. Pediatric head and neck lesions. Ped Clin North Am. 1998; 45(4): 889-905.

9. Weintraub AS, Holzman IR. Neonatal care of infants with head and neck anomalies. Otolaryngol Clin North Am. 2000; 33(6): 1171-89.

10. Filston HC. Hemangiomas, cystic hygromas, and teratomas of the head and neck. Semin Pediatr Surg. 1994; 3(3): 147-159.

11. Pinto A. Pediatric soft tissue lesions. Dent Clin North Am. 2005; 49: 241-58.

12. Kennedy TL, Whitaker M, Pellitteri P, Wood WE. Cystic hygroma/linphangioma: a rational approach to management. Laryngoscope. 2001; 111: 1929-37.

13. Oliveira Olímpio H, Bustorff-Silva J, Gonc As Oliveira Filho A, Cursino de Araujo K. Cross-sectional study comparing different therapeutic modalities for cystic lymphangiomas in children. Clinics 2014; 69(8): 505-508. 
14. Dehner LP. Soft tissue, peritoneum and retroperitoneum. En: Pediatric surgical pathology. Baltimore: Williams and Wilkins, 1987; 869-938.

15. Rarmand M, Kuttenberg J. A new therapeutic concept for the treatment of cystic hygroma. Oral Surg Oral Med Oral Pathol Oral Radiol Endod. 1996; 81(4): 389-395.

16. Fundora MI, Javech GC, Saldivar GO, Oliva RJ. Higroma quístico del cuello: correlación ultrasonográfica y hallazgos morfológicos en quince casos. Rev Cuba Obstet Ginecol. 1999; 15(1): 101-105.

17. Chandrasekaran A. Neonatal solid tumors Pediatrics and Neonatology. 2017, http://dx.doi.org/10.1016/j.pedneo.2016.12.007. 18. Orvidas L, Kasperbauer J. Pediatric lymphangiomas of the head and neck. Ann Otol Rhinol Laryngol. 2000; 109: 411-421.; Philips HE, McGahan JP. Intrauterine fetal cystic hygromas: sonographic detection. Am J Roentgenol. 1981; 136: 799-802. 19. Brennan TD, Miller AS, Chen SY. Lymphangiomas of the oral cavity: A clinicopathologic, immunohistochemical, and electron-microscopic study. J Oral Maxillofac Surg. 1997; 55: 932-5.

20. Beziat JL, Seguin P, Freidel M. Parotidectomy in children. A propos of a homogeneous series of 15 cases. Rev Stomatol Chir Maxillofac 1988; 89: 142-147.

21. Shankargouda P, Roopa SR, Barnali M, Mohammed J, Mahesh M, Anil S. Oral Lesions in Neonates. International Journal of Clinical Pediatric Dentistry, April-June 2016; 9(2): 131-138.

22. Gross E, Sichel J. Congenital neck lesions. Surg Clin North Am. 2006; 86: 383-392, Charabi B, Bretlau P, Bille M, Holmelund M. Cystic hygroma of the head and neck: a long term follow-up of 44 cases. Acta Otolaryngol. 2000; 543: $248-250$.

23. Bracho E, Reyes R, Tovilla JM. Factores de riesgo para recidiva postquirúrgica de linfangiomas en niños. Bol Med Hosp Infant Méx. 2002; 59: 274-281.

24. Orvidas LJ, Kasperbauer JL. Pediatric lymphangiomas of the head and neck. Ann Otol Rhinol Laryngol. $2000 ; 109: 411-21$. 25. Oliveira Olímpio H, Bustorff-Silva J, Gonc A, Oliveira Filho A, Cursino de Araujo K. Cross-sectional study comparing different therapeutic modalities for cystic lymphangiomas in children. Clinics 2014; 69(8): 505-508.

26. Farnoosh S, Don D, Koempel J, Panossian A, Anselmo D, Stanley P. Efficacy of doxycycline and sodium tetradecyl sulfate sclerotherapy in pediatric head and neck lymphatic malformations. International Journal of Pediatric Otorhinolaryngology 79 (2015) 883-887.

27. Ryoma Y, Moriya Y, Okamoto M, Kanaya I, Saito M, Sato M: Biological effect of OK-432 (Picibanil) and possible application to dendritic cell therapy. Anticancer Res. 2004; 24(5C): 3295-301.

Recibido: 02/07/2017

Aceptado: 11/10/2017

Correspondencia: Christian O. Mosca: moscach@yahoo.com M. T. de Alvear 2142. Cátedra de Microbiología y Parasitología. Facultad de Odontología. Universidad de Buenos Aires. Telefono: (5411) 4671-4357/8133 